\title{
Prognostic Value of Plasma Galectin-3 Levels in Patients With Coronary Heart Disease and Chronic Heart Failure
}

\author{
Xiangdong Yu, ${ }^{1} \mathrm{MS}$, Yuemin Sun, ${ }^{1} \mathrm{MD}$, Ying ZhaO, ${ }^{1} \mathrm{MS}$, Wenjuan Zhang, ${ }^{1} \mathrm{MS}$, \\ Zhenwen YANG, ${ }^{1} \mathrm{MD}$, Yuxia GAO, ${ }^{1} \mathrm{MD}$, Heng CAI, ${ }^{1} \mathrm{MD}$, Yongle LI, ${ }^{1} \mathrm{MD}$, \\ Qing WANG, ${ }^{1} \mathrm{MD}$, Bo BIAN, ${ }^{1} \mathrm{MD}$, and Jing NIE, ${ }^{1} \mathrm{MD}$
}

\begin{abstract}
SUMMARY
In this study, we evaluated the prognostic value of plasma galectin-3 levels in patients with coronary heart disease (CHD) and chronic heart failure (HF) and selected 261 CHD patients who were consecutively admitted to our hospital. The enrolled chronic HF patients included HF patients with preserved ejection fraction (HFpEF) and reduced ejection fraction (HFrEF). Patients without HF served as the control group. Galectin-3 and B-type natriuretic peptide (BNP) levels were determined and the primary endpoint was the composite of all-cause mortality and rehospitalization with 12-month follow-up. Plasma galectin-3 levels were higher in HF patients compared with non-HF patients $(P<0.001)$. Receiver operating characteristic (ROC) analyses for diagnosis of HF showed that galectin-3 had the greatest area under the curve (AUC) of $0.756(P<0.001)$, with an optimal cutoff of $10.8 \mathrm{ng} / \mathrm{mL}$, yielding a sensitivity of $81.7 \%$ and a specificity of $61.7 \%$. Follow-up ROC analyses of galectin-3 for outcome prediction showed an optimal cutoff of $17.8 \mathrm{ng} /$ $\mathrm{mL}$, yielding a sensitivity of $97.3 \%$ and a specificity of $77.6 \%$. Galectin-3 yielded an AUC of $0.899(P<0.001)$, whereas the AUC of BNP was $0.633(P=0.022)$. Galectin-3 led to an AUC of $0.931(P<0.001)$ for HFpEF and an AUC of 0.882 $(P<0.001)$ for HFrEF. Cox proportional hazards regression analysis revealed that galectin-3 was an independent prognostic predictor for chronic HF, especially for HFpEF patients (RR: 1.231, 95\% CI: 1.066-1.442). In summary, plasma galectin-3 levels were increased in CHD HF patients and were an independent predictor of all-cause mortality and rehospitalization. In HFpEF patients galectin-3 levels correlated stronger with outcomes than in HFrEF patients. (Int Heart J 2015; 56: 314-318)
\end{abstract}

Key words: Independent predictor, Outcome, Brain natriuretic peptide, Prognosis

$\mathrm{C}$ hronic heart failure (HF) is the end-stage of cardiovascular diseases and despite improvements in medical therapies, the outcomes remain poor with a 5-year mortality rate approaching $50 \%$ in symptomatic patients. HF with preserved ejection fraction (HFpEF) has a similar prognosis as $\mathrm{HF}$ with reduced ejection fraction (HFrEF). ${ }^{1)}$ Brain natriuretic peptide (BNP) has been shown to be a powerful marker for use in the diagnosis and prognosis of HF. Its expression is rapidly unregulated when cardiomyocytes are stretched, but serum concentrations are influenced by other factors including age, renal function and anemia. ${ }^{2}$ Aside from myocardial stretch, other mechanisms, such as inflammation or cardiac remodeling, also play a role in $\mathrm{HF}^{3)}$ but these processes might not be reflected by plasma BNP levels. As a result, the search for new biomarkers, which might reveal other disease processes and be of additional and independent prognostic value, has continued. The $\beta$-galactoside binding galectin- 3 is secreted by activated macrophages. It plays an important regulatory role in cardiac fibrosis and increased expression of collagens, which are key factors for the development heart hypertrophy and progression of $\mathrm{HF}^{4)}$ The up-regulation of myocardial galectin-3 has initially been demonstrated in a rat model of HF-prone hypertensive hearts, ${ }^{5}$ but the prognostic value of plasma galectin-3 levels for $\mathrm{HF}$ patients is still under debate. ${ }^{6-13)}$ Because the majority of previous studies included HFrEF patients only, ${ }^{14)}$ limited data are available for HFpEF individuals and the prognostic value of galectin-3 in patients with coronary heart disease (CHD) and chronic HF has not been fully elucidated. Therefore, this study aimed to investigate the prognostic value of plasma galectin-3 levels in CHD patients with $\mathrm{HFrEF}$ or $\mathrm{HFpEF}$ and to compare the results with BNP, which is an established HF biomarker.

\section{Methods}

Study participants: This prospective study included 261 patients with CHD who were consecutively admitted to the Department of Cardiology, Tianjin Medical University General Hospital from May 2010 to May 2011. A total of 150 chronic HF patients including $48 \mathrm{HFpEF}$ and $102 \mathrm{HFrEF}$ patients were divided into the HF group. The non-HF group consisted of 111

From the ${ }^{1}$ Department of Cardiology, Tianjin Medical University General Hospital, Tianjin, P.R. China

Address for correspondence: Yuemin Sun, MD, Department of Cardiology, Tianjin Medical University General Hospital, 154, Anshan Road, Heping District, Tianjin 300052, P.R. China. E-mail: sunym140818@163.com

Received for publication September 26, 2014. Revised and accepted December 5, 2014.

Released in advance online on J-STAGE April 23, 2015.

All rights reserved by the International Heart Journal Association. 
patients without HF. Patients with a history of myocardial infarction or at least $50 \%$ stenosis in at least one epicardial coronary artery documented by angiography were diagnosed as CHD cases. HF was diagnosed according to ESC Guidelines for the diagnosis and treatment of acute and chronic heart failure 2008. ${ }^{15)}$ Patients with malignant tumors, pulmonary fibrosis, cirrhosis, bronchial asthma, or acute myocardial infarction were excluded. Written informed consent was obtained from all patients and the study was approved by the Chinese Ethics Committee of Registering Clinical Trials and conducted in accordance with the Helsinki Declaration of 1971, as revised in 1983.

Blood collection: A total of $10 \mathrm{~mL}$ fasting blood was drawn from the median cubital vein of all patients at the time of admission and $3 \mathrm{~mL}$ was transferred into chilled disposable tubes containing EDTA and centrifuged at 2,500 rpm for 20 minutes. The plasma was then transferred into $1 \mathrm{~mL}$ cryotubes and stored at $-80^{\circ} \mathrm{C}$ for galectin- 3 analyses. The other $7 \mathrm{~mL}$ of fasting blood samples were used to measure glucose (GLU), hemoglobin $(\mathrm{Hb})$, total cholesterol (TG), triglycerides (TG), low density lipoprotein cholesterol (LDL-c), and BNP.

Measurements of galectin-3 and BNP: The galectin-3 levels were measured using an enzyme-linked immunosorbent assay (ELISA) (BG Medicine, Inc., Waltham, MA, USA). The concentrations of BNP were measured with a Microparticle Enzyme Immunoassay (MEIA) (Abbott Laboratories, Abbott Park, IL, USA). The between-run coefficients of variation (CVs) were $6.3 \%$ at $95 \mathrm{pg} / \mathrm{mL}$ and $4.7 \%$ at $1587 \mathrm{pg} / \mathrm{mL}$ for the BNP assay. The estimated glomerular filtration rate (eGFR), as an indicator of renal function, was calculated using the standard 4-variable Modification of Diet in Renal Disease Group equation. ${ }^{16)}$ Other blood parameters were measured with standard hospital equipment.

Follow-up: The patients were contacted by telephone every 2 months. The primary endpoint was a composite of all-cause mortality and rehospitalization due to HF during 12-month follow-up. The patients who were re-hospitalized and died during the follow-up period were counted as deceased. If multiple re-hospitalizations for one patient occurred, they were recorded as one event.

Statistical analyzes: Continuous variables were tested for nor- mal distribution using the Kolmogorov-Smirnov test, and the results are presented as the mean (standard deviation, SD) for continuous variables based upon their normally distributed data or as the median (interquartile range, p25th-p75th) for continuous non-normally distributed data, and as the number $(\%)$ in categorized variables. The intergroup differences were tested using the Student $t$ test for continuous normally distributed variables, chi-square test for categorical data, and the Wilcoxon rank-sum test for continuous non-normally distributed data. The association between galectin-3 levels and the instantaneous relative risk of death or re-hospitalization was analyzed by Cox proportional hazards regression analysis. To assess the prognostic value of galetin-3, areas under the curve (AUCs) of receiver operating characteristics (ROC) curves were evaluated. Optimal cut-off points were calculated using ROC curves. All tests were 2 -sided and a $P$ value $<0.05$ was considered statistically significant. All statistical analyses were performed using SPSS for Windows (Version 18.0., SPSS Inc., Chicago).

\section{RESULTS}

Baseline characteristics: Compared to non-HF patients, HF patients were more likely to have a lower body mass index (BMI) in addition to lower eGFR and lower hemoglobin values $(P<0.001)$. There was no significant difference in blood pressure, blood glucose, lipid levels, or gender between the two groups. Serum galectin-3 and BNP levels were higher in $\mathrm{HF}$ than in non-HF patients $(P<0.001)$ (Table I).

HFpEF patients were on average older, more likely to be anaemic and hypertensive, with higher BMI and percentage of atrial fibrillation as well as belonging to lower New York Heart Association (NYHA) functional classifications and with a lower proportion of men than HFrEF patients $(P<0.05)$. No difference was observed in the percentages of diabetes mellitus and renal dysfunction between the two groups. Serum BNP levels were higher in HFrEF than in HFpEF cases $(P=0.001)$, but there was no significant difference in galectin-3 levels between the two groups $(P=0.336)$ (Table II).

Diagnostic value of galectin-3 for HF: ROC curves were plot-

Table I. Comparisons of Baseline Characteristics and Clinical Data in HF and Non-HF Patients.

\begin{tabular}{lcrl}
\hline Variable & $\begin{array}{c}\text { HF group } \\
(n=150)\end{array}$ & $\begin{array}{c}\text { non-HF group } \\
(n=111)\end{array}$ & $P$ \\
\hline Age $($ years $)$ & $73 \pm 8$ & $66 \pm 9$ & $<0.001$ \\
Men $(n, \%)$ & 78,52 & 50,55 & 0.707 \\
eGFR $\left(\mathrm{mL} /\right.$ minute $\left.1.73 \mathrm{~m}^{2}\right)$ & $97.4 \pm 25.8$ & $<0.001$ \\
BMI $\left(\mathrm{kg} / \mathrm{m}^{2}\right)$ & $71.7 \pm 31.1$ & $25.9 \pm 4.51$ & 0.001 \\
GLU $(\mathrm{mmol} / \mathrm{L})$ & $23.0 \pm 7.25$ & $6.53 \pm 2.07$ & 0.065 \\
Hb $(\mathrm{g} / \mathrm{L})$ & $7.11 \pm 2.62$ & $135 \pm 16.6$ & $<0.001$ \\
TC $(\mathrm{mmol} / \mathrm{L})$ & $123 \pm 15.8$ & $4.64 \pm 1.15$ & 0.952 \\
TG $(\mathrm{mmol} / \mathrm{L})$ & $4.65 \pm 1.22$ & $1.69 \pm 1.34$ & 0.152 \\
LDL-c $(\mathrm{mmol} / \mathrm{L})$ & $1.49 \pm 0.95$ & $2.75 \pm 0.98$ & 0.481 \\
SBP $(\mathrm{mmHg})$ & $2.84 \pm 1.02$ & $124.56 \pm 24.17$ & 0.050 \\
DBP $(\mathrm{mmHg})$ & $131 \pm 49.5$ & $78.75 \pm 18.27$ & 0.782 \\
Galectin-3 (ng/mL, median,IQR) & $78.9 \pm 22.6$ & $9.3(6.1-13.3)$ & $<0.001$ \\
BNP $(\mathrm{pg} / \mathrm{mL}$, median,IQR) & $18.17(12.08-21.49)$ & $31.7(13.8-85.2)$ & $<0.001$ \\
\hline
\end{tabular}

eGFR indicates estimated glomerular filtration rate; BMI, body mass index; Hb, hemoglobin; TC, total cholesterol; TG, triglycerides; LDL-c, low-density lipoprotein cholesterol; SBP, systolic blood pressure; DBP, diastolic blood pressure; IQR, interquartile range; and BNP, brain natriuretic peptide. 
Table II. Comparisons of Baseline Characteristics and Clinical Data From HFpEF and HFrEF Patients

\begin{tabular}{lccr}
\hline Variable & $\begin{array}{c}\text { HFpEF } \\
(n=48)\end{array}$ & $\begin{array}{c}\text { HFrEF } \\
(n=102)\end{array}$ & $P$ \\
\hline Age (years) & $75 \pm 7$ & $72 \pm 8$ & 0.047 \\
Men (\%) & 45.2 & 69.3 & 0.001 \\
Anaemia (\%) & 60.5 & 47.7 & 0.004 \\
BMI (kg/m ${ }^{2}$ ) & $24.9 \pm 4.36$ & $23.9 \pm 4.28$ & 0.023 \\
Hypertension (\%) & 69.6 & 52.1 & 0.045 \\
Diabetes mellitus (\%) & 33.3 & 48.0 & 0.112 \\
Atrial fibrillation (\%) & 27.1 & 6.86 & 0.001 \\
Mild renal insufficiency (\%) & 38.0 & 36.6 & 0.417 \\
Moderate renal impairment (\%) & 25.1 & 32.4 & 0.052 \\
End-stage renal disease (\%) & 9.70 & 8.40 & 0.357 \\
SBP (mmHg) & $136 \pm 52.8$ & $725 \pm 46.1$ & 0.032 \\
DBP (mmHg) & $82.1 \pm 25.6$ & $2.97 \pm 0.85$ & 0.041 \\
NYHA functional class & $2.63 \pm 0.76$ & $18.64(12.08-21.71)$ & 0.018 \\
Galectin-3 (ng/mL, median, IQR) & $17.45(11.24-21.02)$ & $993.0(598.5-1605.0)$ & 0.336 \\
BNP (pg/mL, median, IQR) & $492.0(323.0-647.5)$ & 0.001 \\
\hline
\end{tabular}

NYHA indicates New York Heart Association; Mild renal insufficiency, $90>$ eGFR $\geq 60 \mathrm{~mL} /$ minute $/ 1.73 \mathrm{~m}^{2}$; Moderate renal impairment, $60>$ eGFR $\geq 30 \mathrm{~mL} /$ minute $/ 1.73 \mathrm{~m}^{2}$; End-stage renal disease, eGFR $<30 \mathrm{~mL} / \mathrm{minute} / 1.73 \mathrm{~m}^{2}$.

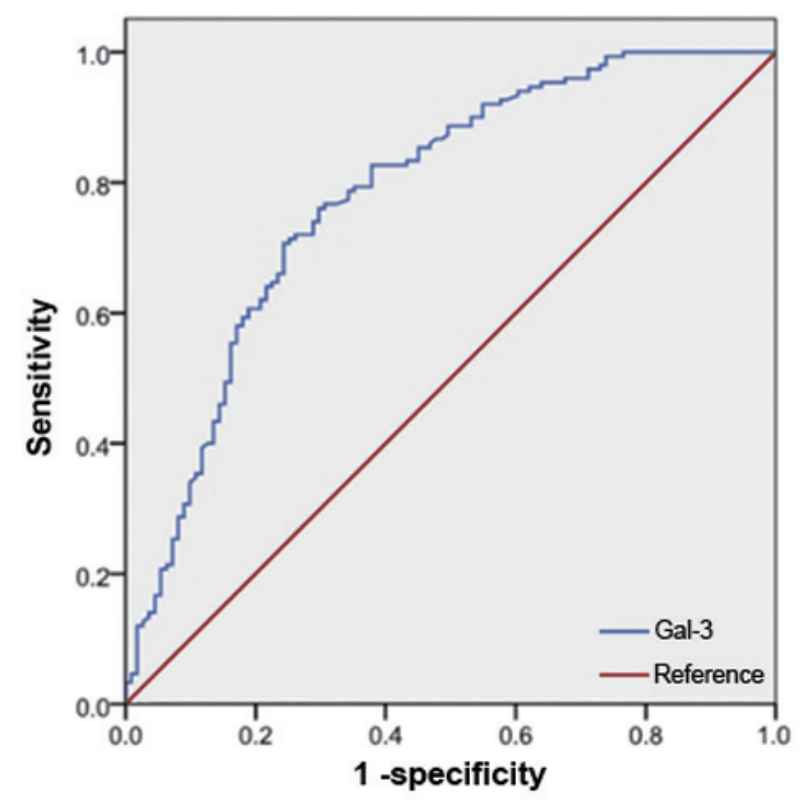

Figure 1. Combined receiver-operating characteristic (ROC) curves of galectin-3 for $\mathrm{HF}$ diagnosis. Galectin-3 AUC $=0.756, P<0.001$.

ted according to the data of $150 \mathrm{HF}$ and 111 non-HF patients; galectin-3 had the greatest area under the curve (AUC) of 0.756 $(P<0.001)$, with an optimal cutoff of $10.8 \mathrm{ng} / \mathrm{mL}$, yielding a sensitivity of $81.7 \%$ and a specificity of $61.7 \%$ (Figure 1).

Prognostic values of galectin-3 and BNP for HF: During the 12-month follow-up period, 37 patients died and 21 were rehospitalized due to worsened HF. The ROC analysis of galectin-3 for the prediction of outcome showed an optimal cutoff of $17.78 \mathrm{ng} / \mathrm{mL}$, yielding a sensitivity of $97.3 \%$ and a specificity of $77.6 \%$. Galectin-3 had an AUC of $0.899(P<0.001)$, whereas the AUC of BNP was $0.633(P=0.022)$ (Figure 2). Galectin-3 had an AUC of 0.931 for HFpEF patients $(P<$ $0.001)$ and 0.882 for HFrEF patients $(P<0.001)$.

In the HFpEF patients, there were 11 deaths and 7 rehospitalizations due to worsened HF. Cox proportional hazards

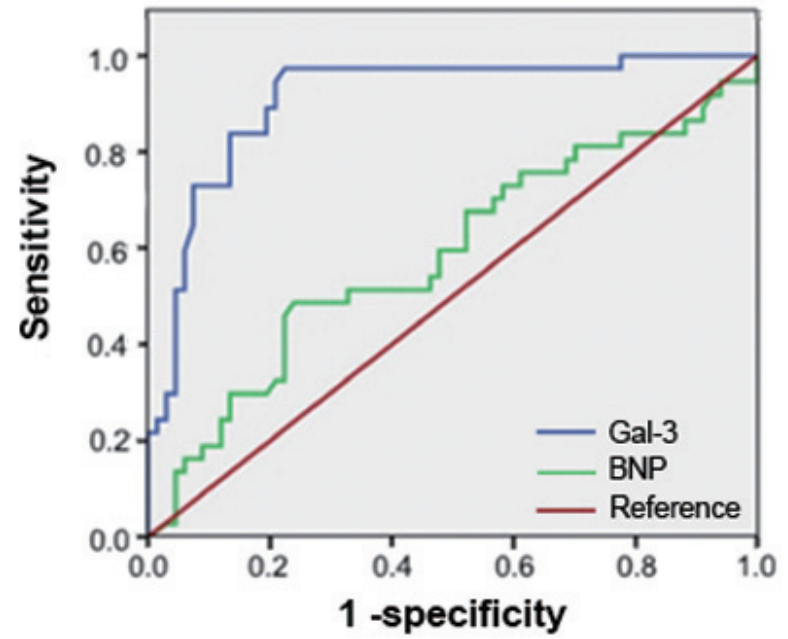

Figure 2. Combined receiver-operating characteristic (ROC) curves of brain natriuretic peptide (BNP) and galectin-3 for death and HF readmission for $\mathrm{HF}$ prognosis for $\mathrm{HF}$ patients after 12 months follow-up. Galectin-3 AUC $=0.899, P<0.001 ; \mathrm{BNP}$ AUC $=0.633, P=0.022$.

regression analysis demonstrated that galectin-3 (RR: 1.231, 95\% CI: 1.066-1.442) and eGFR (RR: $0.933,95 \%$ CI: $0.883-$ $0.987)$ were significant independent predictors for all-cause mortality and rehospitalization due to HF (Table III).

Among patients with HFrEF, there were 26 deaths and 14 re-hospitalizations due to worsened HF. Cox proportional hazards regression analysis showed that BNP (RR: 1.001, 95\% CI: 1.000-1.001) was a significant independent predictor for the primary endpoint, whereas galectin-3 was only a weak predictor (RR: 1.024, 95\% CI: 1.000-1.049) (Table IV).

\section{Discussion}

Since Sharma and colleagues reported the finding that macrophage-derived mediator galectin-3 upregulation induced cardiac fibroblast proliferation, collagen deposition and ven- 
Table III. Cox Proportional Hazards Regression Analysis of HFpEF Patients

\begin{tabular}{lrccl}
\hline Predictor & \multicolumn{1}{c}{$\mathrm{B}$} & $\mathrm{RR}$ & \multicolumn{1}{c}{$P$} & \multicolumn{1}{c}{$95 \% \mathrm{CI}$} \\
\hline Galectin-3 & 0.208 & 1.231 & 0.005 & $1.066 \sim 1.422$ \\
eGFR & -0.069 & 0.933 & 0.015 & $0.883 \sim 0.987$ \\
NYHA functional class & 1.018 & 2.768 & 0.14 & $0.717 \sim 10.681$ \\
Hb & -0.032 & 0.974 & 0.269 & $0.916 \sim 1.025$ \\
BNP & 0.000 & 1.000 & 0.361 & $0.999 \sim 1.001$ \\
\hline
\end{tabular}

$\mathrm{CI}$ indicates confidence interval; $\mathrm{RR}$, relative risk; $\mathrm{B}$, regression coefficient; and $\mathrm{Hb}$, hemoglobin.

tricular dysfunction in a failure-prone hypertrophied rat heart model, galectin-3 has been the subject of HF related research. ${ }^{5)}$ Later, an increased concentration of galectin-3 was found in patients with chronic HF, regardless of etiology and HF typology ${ }^{17)}$ and galectin-3 has also been noted to be significantly up-regulated in the hypertrophied hearts of patients with aortic stenosis and in the plasma of patients with acute and chronic $\mathrm{HF}^{6,8)}$ The median galectin-3 level in our HF cohort was 18.17 $\mathrm{ng} / \mathrm{mL}$, which was higher than that of non-HF patients $(9.31$ $\mathrm{ng} / \mathrm{mL}$ ) and similar to the value of $20.0 \mathrm{ng} / \mathrm{mL}$ reported in the Coordinating study evaluating outcomes of Advising and Counseling in Heart failure $(\mathrm{COACH})$ study ${ }^{18)}$ and the value of $17.6 \mathrm{ng} / \mathrm{mL}$ in the Deventer-Alkmaar heart failure (DEAL-HF) study, ${ }^{8)}$ but higher than concentrations noted by the Heart Failure: A Controlled Trial Investigating Outcomes of Exercise Training (HF-ACTION) study ${ }^{11)}(14.0 \mathrm{ng} / \mathrm{mL})$. The BNP levels of our study participants were essentially higher in HF than in non-HF individuals which is in accordance with previous literature ${ }^{19)}$ and they were higher in HFrEF than in HFpEF patients, which is in agreement with a previous report. ${ }^{18)}$ The relatively lower natriuretic peptide levels suggest a lower diastolic wall stress in HFpEF compared with HFrEF patients.

Diagnostic value of galectin-3: Van Kimmenade, et $a l^{6}$ studied 599 patients presenting with acute dyspnea at the emergency department, 35\% of whom had acute HF. ROC analysis evaluating galectin-3 diagnostic accuracy showed an AUC of 0.72 and values $>6.88 \mathrm{ng} / \mathrm{mL}$ predicted the diagnosis of $\mathrm{HF}$ with a reasonable sensitivity of $80 \%$, but a poor specificity of $52 \%$, and the NT-proBNP had significantly greater AUC than that of galectin-3. However, we found that galectin-3 had the greatest AUC of $0.756(P<0.001)$, with an optimal cutoff of $10.8 \mathrm{ng} /$ $\mathrm{mL}$, yielding a sensitivity of $81.7 \%$ and a specificity of $61.7 \%$. Remarkably, the AUC of BNP was not significantly greater than that of galectin-3. One explanation for the difference might be that NT-proBNP and BNP assays cannot be compared, although the study by Lainchbury, et $a l^{20)}$ found identical areas under the ROC curves ( 0.89 for both), demonstrating the comparability of the two assays. Another explanation might be that the median galectin-3 level $(18.17 \mathrm{ng} / \mathrm{mL})$ in our study was higher than that in the study of Van Kimmenade, et al $(9.2 \mathrm{ng} / \mathrm{mL})$. Additionally, we studied chronic HF patients and the diagnostic performance of galectin-3 may differ from acute HF.

Prognostic value of galectin-3: Up to now there is no consensus concerning the prognostic value of galectin-3. In the DEAL-HF study, ${ }^{8)} 232$ elderly subjects with advanced symptoms from chronic HF were followed for 6.5 years and elevated galectin-3 was found to be a significant predictor of mortality risk even following adjustment for age, eGFR, and NT-
Table IV. Cox Proportional Hazards Regression Analysis of HFrEF Patients

\begin{tabular}{lrccc}
\hline Predictor & \multicolumn{1}{c}{$\mathrm{B}$} & $\mathrm{RR}$ & $P$ & $95 \% \mathrm{CI}$ \\
\hline Galectin-3 & 0.024 & 1.024 & 0.050 & $1.000 \sim 1.049$ \\
eGFR & -0.013 & 0.987 & 0.193 & $0.968 \sim 1.007$ \\
NYHA functional class & 0.297 & 1.346 & 0.328 & $0.742 \sim 2.443$ \\
Hb & -0.020 & 0.980 & 0.107 & $0.956 \sim 1.004$ \\
BNP & 0.001 & 1.001 & 0.001 & $1.000 \sim 1.001$ \\
Age & 0.037 & 1.038 & 0.110 & $0.992 \sim 1.086$ \\
\hline
\end{tabular}

NYHA indicates New York Heart Association; Hb, hemoglobin; and BNP, brain natriuretic peptide.

proBNP, which was confirmed in a recent study. ${ }^{9)}$ The study of chronic systolic HF patients by Tang, et al ${ }^{10)}$ showed higher plasma galectin-3 levels predicted increased risk of all-cause mortality. However, galectin-3 did not predict the composite endpoint of all-cause mortality including cardiac transplantation or HF hospitalization. In the HF-ACTION study, ${ }^{11)}$ in a multivariable modelling and univariate analysis, galectin-3 was not a significant predictor of cardiovascular caused death or hospitalization after addition of NT-proBNP (HR 0.97; $P=$ 0.36 ) and for all-cause mortality, galectin-3 was not a significant factor after further adjustment for NT-proBNP (adjusted HR 1.06; $P=0.30$ ). In addition, the Controlled Rosuvastatin Multinational Trial in Heart Failure (CORONA) study ${ }^{12)}$ demonstrated that galectin-3 is not associated with outcomes of older patients with advanced chronic systolic HF of ischemic etiology and the Val-HeFT study ${ }^{13)}$ subgroup analysis indicated, that though baseline levels of galectin-3 were significantly associated with the risk of death, first morbid event and hospitalization for HF in unadjusted models, the associations were greatly reduced and no longer significant upon addition of other known prognostic variables that were correlated with galectin-3 including NT-proBNP and eGFR.

We evaluated the prognostic value of baseline galectin-3 levels and the results were in agreement with those of previous studies (de Boer, et al, 2011; Lok, et al, 2010); galectin-3 had an AUC of 0.899, with an optimal cutoff of $17.78 \mathrm{ng} / \mathrm{mL}$, yielding a sensitivity of $97.3 \%$ and a specificity of $77.6 \%$, whereas the AUC of BNP was 0.633. The independent prognostic impact of galectin-3 (RR: 1.024, 95\% CI: 1.000-1.049) was attenuated by BNP (RR: 1.001, 95\% CI: 1.000-1.001) in HFrEF patients. De Boer and colleagues studied 592 acute decompensated HF patients in the COACH study ${ }^{18)}$ and showed that galectin-3 had an independent prognostic value even after correction for established risk factors of poor outcome, including age, sex, BNP, renal function, and diabetes mellitus. A recent study, ${ }^{21)}$ in which 419 patients with HF with preserved or mildly reduced left ventricular ejection fraction (LVEF) (LVEF $\geq 45 \%$ ) were enrolled, found that galectin-3 increased independently 1.5 -fold the risk of all-cause mortality and HF readmissions. We found that galectin-3 (RR: $1.231,95 \% \mathrm{CI}$ : 1.066-1.442) and eGFR (RR: 0.933, 95\% CI: 0.883-0.987) were significant independent predictors for all-cause mortality and rehospitalization due to $\mathrm{HF}$ in $\mathrm{HFpEF}$ patients and galectin-3 had a higher AUC of 0.931 in HFpEF than that of 0.882 in HFrEF. These results suggested that the outcomes of HFpEF patients correlated stronger with galectin-3 levels than in HFrEF patients. Galectin-3 is regarded to be a marker of interstitial fibrosis. Considering the pathophysiology of HFpEF, 
which is characterized by hypertrophy, matrix apposition, and myocardial stiffening, it seems reasonable that a matrix and fibrosis marker like galectin-3 might be a more important prognostic marker for HFpEF than for HFrEF. Generally, HFpEF is more common in elderly, female patients associated with more frequent comorbidities such as hypertension and atrial fibrillation, which was confirmed in our study.

De Boer, et $a l^{18)}$ verified that correction for eGFR resulted in some loss, albeit very small, of predictive galectin-3 power, suggesting that some of the prognostic power of galectin-3 may be mediated via renal functions. Since increased galectin-3 is also associated with renal fibrosis, renal impairment may in part determine the prognostic role of galectin-3 in HF, since renal dysfunction is one of the most powerful predictors of HF prognosis. ${ }^{10)}$

There are limitations in this study. Firstly, the sample size was relatively small, especially for patients with HFpEF. Secondly, we did not include other markers with potential prognostic value in HF, such as GDF-15 or high-sensitivity C-reactive protein (hs-CRP), which might have affected our findings. Thirdly, we did not measure galectin-3 over time which could have influenced the results, since several studies showed that serial increases of galectin-3 were independently associated with outcomes. ${ }^{13,22,23)}$ Finally, the present study population was a rather selected population of $\mathrm{HF}$ patients with $\mathrm{CHD}$, and our findings could not necessarily be extrapolated to the general HF population.

Conclusion: Plasma galectin-3 levels were increased in patients with $\mathrm{CHD}$ and $\mathrm{HF}$ and was an independent predictor of all-cause mortality and rehospitalization due to HF. In patients with HFpEF, galectin-3 levels correlated stronger with prognosis than in HFrEF patients. Additional studies are required to confirm these findings and to determine further the prognostic value of galectin-3 in chronic HF patients.

\section{Disclosures}

There are no conflicts of interest to disclose.

\section{REFERENCES}

1. Owan TE, Hodge DO, Herges RM, Jacobsen SJ, Roger VL, Redfield MM. Trends in prevalence and outcome of heart failure with preserved ejection fraction. N Engl J Med 2006; 355: 251-9.

2. Hogenhuis J, Voors AA, Jaarsma T, et al. Anaemia and renal dysfunction are independently associated with BNP and NT-proBNP levels in patients with heart failure. Eur J Heart Fail 2007; 9: $787-$ 94.

3. Yanavitski M, Givertz MM. Novel biomarkers in acute heart failure. Curr Heart Fail Rep 2011; 8: 206-11. (Review)

4. de Boer RA, Yu L, van Veldhuisen DJ. Galectin-3 in cardiac remodeling and heart failure. Curr Heart Fail Rep 2010; 7: 1-8. (Review)

5. Sharma UC, Pokharel S, van Brakel TJ, et al. Galectin-3 marks activated macrophages in failure-prone hypertrophied hearts and contributes to cardiac dysfunction. Circulation 2004; 110: 3121-8.

6. van Kimmenade RR, Januzzi JL Jr, Ellinor PT, et al. Utility of amino-terminal pro-brain natriuretic peptide, galectin-3, and apelin for the evaluation of patients with acute heart failure. J Am Coll Cardiol 2006; 48: 1217-24.
7. Shah RV, Chen-Tournoux AA, Picard MH, van Kimmenade RR, Januzzi JL. Galectin-3, cardiac structure and function, and longterm mortality in patients with acutely decompensated heart failure. Eur J Heart Fail 2010; 12: 826-32.

8. Lok DJ, Van Der Meer P, de la Porte PW, et al. Prognostic value of galectin-3, a novel marker of fibrosis, in patients with chronic heart failure: data from the DEAL-HF study. Clin Res Cardiol 2010; 99: 323-8.

9. Gruson D, Mancini M, Ahn SA, Rousseau MF. Galectin-3 testing: validity of a novel automated assay in heart failure patients with reduced ejection fraction. Clin Chim Acta 2014; 429: 189-93.

10. Tang WH, Shrestha K, Shao Z, et al. Usefulness of plasma galectin-3 levels in systolic heart failure to predict renal insufficiency and survival. Am J Cardiol 2011; 108: 385-90.

11. Felker GM, Fiuzat M, Shaw LK, et al. Galectin-3 in ambulatory patients with heart failure: results from the HF-ACTION study. Circ Heart Fail 2012; 5: 72-8.

12. Gullestad L, Ueland T, Kjekshus J, et al. The predictive value of galectin-3 for mortality and cardiovascular events in the Controlled Rosuvastatin Multinational Trial in Heart Failure (CORONA). Am Heart J 2012; 164: 878-83.

13. Anand IS, Rector TS, Kuskowski M, Adourian A, Muntendam P, Cohn JN. Baseline and serial measurements of galectin-3 in patients with heart failure: relationship to prognosis and effect of treatment with valsartan in the Val-HeFT. Eur J Heart Fail 2013; 15: 511-8.

14. Braunwald E. Biomarkers in heart failure. N Engl J Med 2008; 358: 2148-59. (Review)

15. Dickstein K, Cohen-Solal A, Filippatos G, et al. ESC Guidelines for the diagnosis and treatment of acute and chronic heart failure 2008: the Task Force for the Diagnosis and Treatment of Acute and Chronic Heart Failure 2008 of the European Society of Cardiology. Developed in collaboration with the Heart Failure Association of the ESC (HFA) and endorsed by the European Society of Intensive Care Medicine (ESICM). Eur Heart J 2008; 29: 2388442. (Review)

16. Levey AS, Bosch JP, Lewis JB, Greene T, Rogers N, Roth D. A more accurate method to estimate glomerular filtration rate from serum creatinine: a new prediction equation. Modification of Diet in Renal Disease Study Group. Ann Intern Med 1999; 130: 46170.

17. Hrynchyshyn N, Jourdain P, Desnos M, Diebold B, Funck F. Galectin-3: a new biomarker for the diagnosis, analysis and prognosis of acute and chronic heart failure. Arch Cardiovasc Dis 2013; 106: 541-6.

18. de Boer RA, Lok DJ, Jaarsma T, et al. Predictive value of plasma galectin-3 levels in heart failure with reduced and preserved ejection fraction. Ann Med 2011; 43: 60-8.

19. Tschöpe C, Kasner M, Westermann D, Gaub R, Poller WC, Schultheiss HP. The role of NT-proBNP in the diagnostics of isolated diastolic dysfunction: correlation with echocardiographic and invasive measurements. Eur Heart J 2005; 26: 2277-84.

20. Lainchbury JG, Campbell E, Frampton CM, Yandle TG, Nicholls MG, Richards AM. Brain natriuretic peptide and n-terminal brain natriuretic peptide in the diagnosis of heart failure in patients with acute shortness of breath. J Am Coll Cardiol 2003; 42: 728-35.

21. Carrasco-Sánchez FJ, Aramburu-Bodas O, Salamanca-Bautista P, et al. Predictive value of serum galectin-3 levels in patients with acute heart failure with preserved ejection fraction. Int $\mathrm{J}$ Cardiol 2013; 169: 177-82.

22. Motiwala SR, Szymonifka J, Belcher A, et al. Serial measurement of galectin-3 in patients with chronic heart failure: results from the ProBNP Outpatient Tailored Chronic Heart Failure Therapy (PROTECT) study. Eur J Heart Fail 2013; 15: 1157-63.

23. van der Velde AR, Gullestad L, Ueland T, et al. Prognostic value of changes in galectin-3 levels over time in patients with heart failure: data from CORONA and COACH. Circ Heart Fail 2013; 6: 219-26. 